\title{
Equilibrium sizes of jellium metal clusters in the stabilized spin-polarized jellium model
}

\author{
M. Payami
}

October 25, 2018

Center for Theoretical Physics and Mathematics, Atomic Energy Organization of Iran, P. O. Box 11365-8486, Tehran, Iran

October 25, 2018

\begin{abstract}
We have used the stabilized spin-polarized jellium model to calculate the equilibrium sizes of metal clusters. Our self-consistent calculations in the local spin-density approximation show that for an $N$-electron cluster, the equilibrium is achieved for a configuration in which the difference in the numbers of up-spin and down-spin electrons is zero or unity, depending on the total number of electrons. That is, a configuration in which the spins are maximally compensated. This maximum spincompensation results in both the alternation in the average distance between the nearest neighbor ions and the odd-even alternations in the ionization energies of alkali metal clusters, in a good agreement with the molecular dynamics findings and the experiment. These suggest a realistic and more accurate method for calculating the properties of metal clusters in the context of jellium model than previous jellium model methods.
\end{abstract}

71.15.Hx, 71.15.Mb, 71.15.Nc 


\section{Introduction}

The simplest model used in theoretical study of the properties of simple metal clusters is jellium model (JM) with spherical geometry. [1, 2, 3] In this model, the ions are replaced by a uniform positive charge background sphere of density $n=3 / 4 \pi r_{s}^{3}$ and radius $R=(z N)^{1 / 3} r_{s}$ where $z, N$ and $r_{s}$ are the valence of the atom, the number of constituent atoms of the cluster and the bulk value of the Wigner-Seitz (WS) radius of the metal, respectively. This model can be useful only when the pseudopotentials of the ions do not significantly affect the electronic structure. But, it is well-known that the JM has some drawbacks. [4, 5] Keeping the simplicity of the JM, the stabilized jellium model (SJM) of Perdew et al. [6] has lifted the essential deficiencies of the JM and significantly improved the results of calculations. In the SJM, the bulk metal (which is spin-unpolarized in the absence of external magnetic field) has been made stabilized through the introduction of a pseudopotential and fixing its core radius by a value that makes the pressure on the unpolarized bulk system to vanish. However, applying the SJM in the framework of rigid jellium background [7] may be suitable for closed-shell clusters of which the spin polarization of the valence electrons vanishes. It is well-known that, for example, the bond-length of a diatomic molecule depends on the relative orientations of the valence electrons. Hence, considering a metal cluster as a large molecule, or the bulk metal as a huge molecule, one should take account of the volume change due to spin polarization. We therefore, expect that the spherical jellium radius should be different for an $N$-atom cluster with two different spin configurations. These facts led us to consider a stabilized jellium model in which the spin degrees of freedom be present. Stabilizing the jellium system with non-zero spin polarization, $\zeta$, for the valence electrons resulted in the stabilized spin-polarized jellium model (SSPJM). [8] Here $\zeta=\left(n_{\uparrow}-n_{\downarrow}\right) /\left(n_{\uparrow}+n_{\downarrow}\right)$ and $n_{\uparrow}, n_{\downarrow}$ are the spin-up and spin-down electron densities, respectively. The SSPJM can be applied to metal clusters in two different ways. The first method, which has been used in Ref. [8], exploits the fact that the bulk metal expands as $\zeta$ increases. We call that method as SSPJM1 throughout this paper. In that method, the jellium sphere radius is taken as $R(\zeta)=(z N)^{1 / 3} \bar{r}_{s}(\zeta)$. For $\bar{r}_{s}(\zeta)$ we had taken $\bar{r}_{s}(\zeta)=\bar{r}_{s}(0)+\Delta r_{s}(\zeta)$ in which $\bar{r}_{s}(0)$ was the observed value of the bulk WS radius of the metal and $\Delta r_{s}$ was obtained by the application of the local spin-density approximation (LSDA) to the infinite electron gas system. However, in our phenomenological accounting of the volume change, the core radius of the pseudo-potential for the electron-ion interaction has been considered as a parameter, which becomes polarization dependent as we force the pressure of the polarized bulk system to vanish. Using that scheme, we had calculated the energies of different metal clusters, both neutral and singly ionized, for different spin configurations 
and had shown that instead of Hund's first rule for the ground state, the maximum spincompensation (MSC) rule was governing. [8] The MSC property which originates here from the polarization dependence of the core radius, leads to the odd-even alternations in the ionization energies that had been observed experimentally in the alkali metal clusters. [9] On the other hand, if one assumes a fixed, polarization-independent form for the electronion interaction, the MSC property will be realized only for non-spherical geometries of the jellium background.

We have recently shown 10 that it is not always necessary for a finite spherical jellium system to increase its size as the polarization, $\zeta$, is increased. This can be explained by considering the fact that for an open-shell cluster if one increases the spin polarization from the possible minimum value consistent with the Pauli exclusion principle, one should make a spin-flip in the last uncomplete shell. Because of high degeneracy for the spherical geometry, this spin-flip in the last shell does not change appreciably the kinetic energy contribution to the total energy but changes appreciably the exchange-correlation energy which in turn gives rise to a deeper effective potential that makes the Kohn-Sham (KS) [11] orbitals more localized and therefore a smaller size for the cluster. On the other hand, although the SSPJM1 results in better ionization energies than the SJM [7] in that it reproduces the odd-even alternation, it always predicts incorrect cluster sizes. That is, in the SSPJM1, the equilibrium $r_{s}$ for a cluster is taken to be greater or equal to the bulk value of $r_{s}$ ( see Fig. 3 of Ref. [8]), so that it approaches the bulk value from the above; whereas, the molecular dynamics (MD) results for the average distance between the nearest neighbor atoms show that the equilibrium $r_{s}$ value of the neutral clusters are less than the bulk value and it approaches the bulk value from the below [ see Fig 15(a) of Ref. 13]. To incorporate this correct behavior into our SSPJM calculations, which is the subject of this paper, we proceed parallel to the work of Perdew et al. 12 for the spinpolarized case and call this method as SSPJM2. In the SSPJM2, for a given polarization, we first obtain the value of the core radius of the pseudopotential that stabilizes the bulk system, say $r_{c}^{B}$, and then, using this value of $r_{c}^{B}$ in the energy functional of the cluster, we change the radius of the jellium sphere until the minimum energy is achieved. Our self-consistent calculations show that the absolute minimum energy corresponds to a spin configuration with maximum compensation as in the SSPJM1 case. The equilibrium $r_{s}$ values corresponding to these minima lie below the bulk value, reproducing the correct behavior. These equilibrium $r_{s}$ values determine the equilibrium sizes of the clusters. If we plot the equilibrium $r_{s}$ value as a function of the number of constituent atoms in an alkali metal cluster, we see an alternating behavior, consistent with the MD results. [13] In this paper we have found the equilibrium properties of neutral and singly ionized Cs, $\mathrm{Na}$ and Al clusters of various sizes $(2 \leq N \leq 42)$ using jellium with sharp boundaries. We 
have also repeated the SSPJM2 calculations using a jellium sphere with diffuse boundary. For the sake of comparison, we have derived the results of the work by Perdew et al. 12. which is denoted by SJM1. Comparing our SSPJM2 results with those of SSPJM1 show that here, the average energies per electron and the ionization energies remain more or less the same but here, our SSPJM2 calculations show an improvement over the SSPJM1 results for the equilibrium sizes of the clusters.

In section 2 the calculational schemes has been explained. Section 3 is devoted to the results of our calculations and finally, we conclude this work in section 4 .

\section{Calculational Scheme}

In the context of the SSPJM, the average energy per valence electron in the bulk with density parameter $r_{s}$ and polarization $\zeta$ is given by [8]

$$
\varepsilon\left(r_{s}, \zeta\right)=t_{s}\left(r_{s}, \zeta\right)+\varepsilon_{x c}\left(r_{s}, \zeta\right)+\bar{w}_{R}\left(r_{s}, r_{c}\right)+\varepsilon_{\mathrm{M}}\left(r_{s}\right),
$$

where $t_{s}$ and $\varepsilon_{x c}$ are noninteracting kinetic energy and exchange-correlation energies per electron, respectively. $\bar{w}_{R}$ is the average value (over the WS cell) of the repulsive part of the Ashcroft empty core 14 pseudopotential,

$$
w(r)=-\frac{2 z}{r}+w_{R}, \quad w_{R}=+\frac{2 z}{r} \theta\left(r_{c}-r\right),
$$

and is given by $\bar{w}_{R}=3 r_{c}^{2} / r_{s}^{3}$. In Eq.(2), $z$ is the valence of the atom, and $\theta(x)$ is the ordinary step function which assumes the value of unity for positive arguments, and zero for negative values. The core radius, $r_{c}$, will be fixed by setting the pressure of the bulk system equal to zero at equilibrium density $\bar{n}(\zeta)=3 / 4 \pi \bar{r}_{s}^{3}(\zeta)$. In Eq.(1), $\varepsilon_{\mathrm{M}}$ is the average Madelung energy, $\varepsilon_{\mathrm{M}}=-9 z / 5 r_{0}$. Here, $r_{0}$ is the radius of the WS sphere, $r_{0}=z^{1 / 3} r_{s}$, and for monovalent metals $z=1$, and for polyvalent metals we set $z^{*}=1$ (for details see Ref.[6]). All equations throughout this paper are expressed in Rydberg atomic units. The bulk stability is achieved when $r_{c}$ takes a value that makes the pressure of the system with a given $\zeta$ to vanish at $r_{s}=\bar{r}_{s}(\zeta)$ :

$$
\left.\frac{\partial}{\partial r_{s}} \varepsilon\left(r_{s}, \zeta, r_{c}\right)\right|_{r_{s}=\bar{r}_{s}(\zeta)}=0 .
$$

The derivative is taken at fixed $\zeta$ and $r_{c}$. Solution of the above equation gives the bulk value of $r_{c}$ as a function of $\bar{r}_{s}$ and $\zeta$. Here, $\bar{r}_{s}(\zeta)$ is the equilibrium density parameter for 
the bulk system with given $\zeta$ and is evaluated by

$$
\bar{r}_{s}(\zeta)=\bar{r}_{s}(0)+\Delta r_{s}^{\mathrm{EG}}(\zeta) .
$$

Here, $\bar{r}_{s}(0)$ takes the observed value for a metal and for $\mathrm{Cs}, \mathrm{Na}$, and $\mathrm{Al}$ it takes the values of 5.63, 3.99, and 2.07, respectively. In the second term of the right hand side, the superscript "EG" refers to the electron gas, and $\Delta r_{s}^{\mathrm{EG}}(\zeta)$ is evaluated by setting the pressure of the electron gas system equal to zero (see Eq.(19) of Ref. [8]). The solution of Eq.(3) at equilibrium density gives the bulk value of the core radius:

$$
r_{c}^{B}\left(\bar{r}_{s}, \zeta\right)=\frac{\bar{r}_{s}^{3 / 2}}{3}\left\{-2 t_{s}\left(\bar{r}_{s}, \zeta\right)-\varepsilon_{x}\left(\bar{r}_{s}, \zeta\right)+\bar{r}_{s}\left(\frac{\partial}{\partial \bar{r}_{s}}\right)_{\zeta} \varepsilon_{c}\left(\bar{r}_{s}, \zeta\right)-\varepsilon_{M}\left(\bar{r}_{s}\right)\right\}^{1 / 2} .
$$

Now, using $r_{c}^{B}$ in the SSPJM energy functional of a cluster [ Eq.(20) of Ref.[\&]], we obtain the SSPJM2 energy as

$$
\begin{aligned}
E_{\mathrm{SSPJM} 2}\left[n_{\uparrow}, n_{\downarrow}, n_{+}\right]= & E_{\mathrm{JM}}\left[n_{\uparrow}, n_{\downarrow}, n_{+}\right]+\left(\varepsilon_{M}\left(r_{s}\right)+\bar{w}_{R}\left(r_{c}^{B}, r_{s}\right)\right) \int d \mathbf{r} n_{+}(\mathbf{r}) \\
& +\langle\delta v\rangle_{\mathrm{WS}}\left(r_{c}^{B}, r_{s}\right) \int d \mathbf{r} \Theta(\mathbf{r})\left[n(\mathbf{r})-n_{+}(\mathbf{r})\right],
\end{aligned}
$$

where

$$
\begin{aligned}
E_{\mathrm{JM}}\left[n_{\uparrow}, n_{\downarrow}, n_{+}\right]= & T_{s}\left[n_{\uparrow}, n_{\downarrow}\right]+E_{x c}\left[n_{\uparrow}, n_{\downarrow}\right] \\
& +\frac{1}{2} \int d \mathbf{r} \phi\left(\left[n, n_{+}\right] ; \mathbf{r}\right)\left[n(\mathbf{r})-n_{+}(\mathbf{r})\right]
\end{aligned}
$$

and

$$
\phi\left(\left[n, n_{+}\right] ; \mathbf{r}\right)=2 \int d \mathbf{r}^{\prime} \frac{\left[n\left(\mathbf{r}^{\prime}\right)-n_{+}\left(\mathbf{r}^{\prime}\right)\right]}{\left|\mathbf{r}-\mathbf{r}^{\prime}\right|} .
$$

In Eq. (6), $\langle\delta v\rangle_{\mathrm{wS}}$ is the average of the difference potential over the Wigner-Seitz cell and the difference potential, $\delta v$, is defined as the difference between the pseudopotential of a lattice of ions and the electrostatic potential of the jellium background.

The first and second terms in the right hand side of Eq.(7) are the non-interacting kinetic energy and the exchange-correlation energy, and the last term is the Coulomb interaction energy of the system. In our spherical JM, we have

$$
n_{+}(\mathbf{r})=\frac{3}{4 \pi r_{s}^{3}} \theta(R-r)
$$


in which $R=(z N)^{1 / 3} r_{s}$ is the radius of the jellium sphere, and $n(\mathbf{r})$ denotes the electron density at point $\mathbf{r}$ in space.

Applying the SSPJM2 to an $N$ electron cluster with $N_{\uparrow}$ up-spin and $N_{\downarrow}$ down-spin electrons $\left(N=N_{\uparrow}+N \downarrow\right)$ and polarization $\zeta=\left(N_{\uparrow}-N_{\downarrow}\right) /\left(N_{\uparrow}+N_{\downarrow}\right)$, the total energy becomes a function of $N, \zeta, r_{s}$, and $r_{c}^{B}$ where $r_{s}$ is the density parameter of the jellium background and $r_{c}^{B}$ is given by Eq.(5). The equilibrium density parameter, $\bar{r}_{s}(N, \zeta)$, for a cluster is the solution of

$$
\left.\frac{\partial}{\partial r_{s}} E\left(N, \zeta, r_{s}, r_{c}^{B}\right)\right|_{r_{s}=\bar{r}_{s}(N, \zeta)}=0 .
$$

Here again, the derivative is taken at fixed values of $N, \zeta$, and $r_{c}^{B}$. For an $N$-electron cluster, we have solved the KS equations 11] self-consistently for various spin configurations and $r_{s}$ values and obtained the absolute minimum-energy spin configuration and its corresponding density parameter.

\section{Results}

We have applied the SSPJM2 to calculate the equilibrium energies and sizes of different metal clusters. In our calculations for an $N$ electron cluster, we have solved the KS equations for all possible spin configurations $0 \leq \zeta \leq 1$ and obtained the minimum values of energies and corresponding $r_{s}$ values of each configuration. The self-consistent calculations for $\mathrm{Cs}$, $\mathrm{Na}$, and $\mathrm{Al}$ with $2 \leq N \leq 42$ show that the absolute minimumenergy configuration obeys the MSC rule and the equilibrium $r_{s}$ value for the cluster, $\bar{r}_{s}(N, \zeta)$, is less than the bulk value because, for small clusters the ratio of surface to volume energies become comparable and the surface tension compresses the cluster. This effect is known as self-compression. 12] In Fig. [1 we have compared the equilibrium $r_{s}$ values of "generic clusters", JM1 (see Ref. 10), with the SJM1 results which reproduce correct trends. [12] To clarify the concept of the "generic cluster", suppose that one solves the self-consistent KS-LSDA equations for a spherical simple JM cluster with jellium radius equal to $R=N^{1 / 3} r_{s}$ and total number of electrons $N$. For a given $N$, these calculations are performed for different $r_{s}$ values as well as different spin configurations until the equilibrium $r_{s}$ value, $\bar{r}_{s}(N, \zeta)$, corresponding to the absolute minimum-energy configuration is obtained ( See Fig. 4 of Ref.10). Since in the calculations one does not use any specific parameter corresponding to a certain metal, the result does not simulate any real cluster, and we call it an $N$-electron "generic cluster". In the limit of $N \rightarrow \infty$, the

infinite generic cluster tends to the electron gas system for which $\zeta \rightarrow 0$ and $\bar{r}_{s} \rightarrow 4$.18. As 
is seen from the figure, the equilibrium $r_{s}$ value for the generic cluster approaches the bulk value, 4.18 , from the above which does not simulate the correct behavior for a real metal cluster. It is seen that for the generic clusters the equilibrium values are greater than the value $r_{s}=4.18$ whereas the SJM1 predicts values that are smaller than the bulk value for $\mathrm{Na}\left(r_{s}=3.99\right)$ in agreement with the MD findings. This comparison clearly shows that simple JM gives wrong molecular bond lengths. We have performed our calculations based on Eq. (10) both for jellium with sharp boundary, SSPJM2, and diffuse jellium, dif-SSPJM2. For our diffuse jellium calculations we have used the background density with the radial dependence as 15

$$
n_{+}(r)=\left\{\begin{array}{l}
n\left\{1-(R+t) e^{-R / t}[\sinh (r / t)] / r\right\}, \quad r \leq R \\
n\left\{1-((R+t) / 2 R)\left(1-e^{-2 R / t}\right)\right\} R e^{(R-r) / t} / r, \quad r>R,
\end{array}\right.
$$

where $n=3 / 4 \pi r_{s}^{3}, R=N^{1 / 3} r_{s}$, and $t$ is a parameter related to the surface thickness. We have chosen $t=1$ in all our diffuse jellium calculations and then have varied the value of $r_{s}$ until the minimum energy is achieved. Figure 目(a) compares the equilibrium $r_{s}$ values of neutral cesium clusters for different sizes. It is seen that in most cases (rather large clusters for which $\zeta<<1$ ), the SSPJM2 and the SJM1 predict the same values for the equilibrium $r_{s}$; whereas for $N=3,5$, and 7 the SSPJM2 predicts larger values. These larger values give rise to an alternation in the plot. The values obtained from the dif-SSPJM2 lie below the values obtained from the SSPJM2 and the SJM1. Figure 2(b) shows the results obtained for singly ionized cesium clusters. In this case, we see that the alternations persist up to $N=15$ and have relatively large amplitudes. For $N=3$ i.e., singly ionized 4-atom cluster, the value obtained from the SSPJM2 has become larger than the bulk value which is related to the rough evaluation of $\Delta r_{s}$. In Fig. 3(a) we have shown the same quantities for neutral Na clusters. The behavior of SSPJM2 results is the same as in Fig. 2(a) but, in the case of the dif-SSPJM2 the value for $N=5$ has become nearly equal to that of $N=6$ and also, the value for $N=3$ is less than that of $N=4$ which completely differs from the SSPJM2 results. Also, we could not find any finite value for $N=2$ case in the dif-SSPJM2. That is, as much as we decrease the input $r_{s}$ value, the total energy correspondingly decreases. This means that the surface tension dominates the internal pressure and collapses the cluster. Of course, this is not the case in reality and it is the consequence of the fact that here the surface thickness, $t$, has become comparable to the cluster radius, $R$. Figure 3(b) compares the plots of average distance between the nearest neighbors obtained from our SSPJM2 calculations and the MD calculations of Röthlisberger and Andreoni. 13 In order to estimate the average distance between the nearest neighbor ions in the cluster, we have assumed a $b c c$ structure as in the bulk of 
Na. Then the shortest distance between the ions, $d$, is related to the lattice constant, $a$, through $d=a \sqrt{3} / 2$. But, in the $b c c$ structure for Na, there are two electrons in a cell

and therefore, $a=2 \sqrt{\pi / 3} r_{s}$. Combining these two relations results in $d=\sqrt{\pi} r_{s}$. The value $r_{s}=3.99$ is appropriate for room temperature $(T=300 \mathrm{~K})$ which results in a value of $d=7.07$ bohrs whereas, for $T=0 \mathrm{~K}$ the appropriate value for $r_{s}$ is 3.93 which gives rise to the value $d=6.96$ bohrs. Therefore, our results should lie above the MD results [ see Fig 15(a) of Ref. 13] because, the MD calculations have been performed for zero temperature. In Fig. 3(c) we have shown the plots of equilibrium $r_{s}$ values for singly ionized sodium clusters as functions of number of electrons, $N$. The behavior is more or less the same as neutral one. Figure 4 (a) compares the SSPJM2 results for neutral Al clusters with the results obtained using the SJM1. Here, we have taken the effective value of $z^{*}=1$. The diamonds and squares in the plot show the physical points. The main difference between our results and the SJM1 is in the size of the jellium atom of Al. In Fig. 因(b) we have compared the results for singly ionized Al clusters. The results show some differences for values of $N$ away from shell closings. Looking at the above-mentioned figures, we note that in all the three cases of $\mathrm{Cs}$, Na, and Al the SSPJM2 results in a larger or equal values for the average distance than the SJM1, and in addition show alternations for small clusters.

Finally, in Fig. 5(a) we have compared the plots of the total energies per electron in the two schemes of the SSPJM2 and the SSPJM1 for Na clusters. It is seen that in the SSPJM2 the energies are relatively lower than those of the SSPJM1 for smaller clusters but the same for larger ones. We have also calculated the ionization energies of the clusters using the dif-SSPJM2 and and compared with the dif-SSPJM1 and experimental values in Fig. 5(b). Here also the odd-even alternations show up themselves in the SSPJM2 as well as in the dif-SSPJM2 results and the values obtained are more or less the same as in the dif-SSPJM1 [see Fig. 7(c) of Ref. 8]. Therefore, the SSPJM2 calculations for simple metal clusters has improved the previous work, SSPJM1, in that it not only reproduces the odd-even alternations in the ionization energies, but also it gives correct behavior for the equilibrium sizes of the clusters.

\section{Summary and Conclusion}

In this work we have performed the SSPJM calculations as in the case of ab initio molecular structure calculations. That is, we have firstly calculated the stabilizing core radius of the pseudopotential, $r_{c}^{B}$, for the bulk system with nonzero spin polarization. Then, using this value in the energy functional of a cluster with given values of $N$ and $\zeta$, the energy 
becomes a function of the single variable $r_{s}$, the density parameter of the uniform jellium background. Minimizing this function with respect to $r_{s}$ gives us the equilibrium $r_{s}$ value and energy of the cluster with that specified $N$ and $\zeta$. Our self-consistent KSLSDA calculations show that the equilibrium configuration is one in which the spins are maximally compensated as in our previous findings. [8] This maximum spin compensation gives rise to the odd-even alternations seen in the experimental ionization energy plot of alkali metal clusters. Calculating the average distance between the nearest neighbors of Na clusters, we find a good agreement between our SSPJM2 results and those obtained from MD calculations. We have therefore improved our previous SSPJM1 results in that the odd-even property is kept the same as before but here, the sizes of the smaller clusters have been predicted correctly.

\section{Acknowledgements}

The author would like to thank John P. Perdew for reading the manuscript and the useful discussions on the subject. He also acknowledges Bahram Payami for providing computer facilities. 


\section{References}

[1] W. E. Ekardt, Phys. Rev. B 29, 1558 (1984).

[2] W. D. Knight, K. Clemenger, W. A. de Heer, W. A. Saunders, M. Y. Chou, and M. L. Cohen, Phys. Rev. Lett. 52, 2141 (1984).

[3] M. Brack, Rev. Mod. Phys. 65, 677 (1993), and references therein.

[4] N. D. Lang and W. Kohn, Phys. Rev. B 1, 4555 (1970).

[5] N. W. Ashcroft and D. C. Langreth, Phys. Rev. 155, 682 (1967).

[6] J. P. Perdew, H. Q. Tran, and E. D. Smith, Phys. Rev. B 42, 11627 (1990).

[7] M. Brajczewska, C. Fiolhais, and J. P. Perdew, Int. J. Quantum Chem., Quantum Chem. Symp. 27, 249 (1993). We have calculated the ionization energies and plotted the resuls for Na clusters in Fig. 7(c) of Ref. [8].

[8] M. Payami and N. Nafari, J. Chem. Phys. 109, 5730 (1998).

[9] See Fig. 26 in W. A. de Heer, Rev. Mod. Phys. 65, 611 (1993), and references therein.

[10] M. Payami, J. Chem. Phys. 111, 8344 (1999).

[11] W. Kohn and L. J. Sham, Phys. Rev. 140, A1133 (1965).

[12] J. P. Perdew, M. Brajczewska, and C. Fiolhais, Solid State Commun. 88, 795 (1993).

[13] U. Röthlisberger and W. Andereoni, J. Chem. Phys. 94, 8129 (1991).

[14] N. W. Ashcroft, Phys. Lett. 23, 48 (1966).

[15] A. Rubio, L. C. Balbás, and J. A. Alonso, Z. Phys. D19, 93 (1991). 
Figure 1: The equilibrium $r_{s}$ values in atomic units as functions of the cluster size $N$. The solid squares correspond to the "generic clusters" (JM1) defined in the text and the large squares correspond to Na clusters using the method of Ref.12. The dashed and solid lines correspond to the equilibrium $r_{s}$ value of the bulk (4.18) in simple JM and to the bulk value of sodium (3.99), respectively.

Figure 2: (a) The equilibrium $r_{s}$ values in atomic units as functions of the cluster size for cesium clusters obtained from using the three schems of SJM1, SSPJM2, and dif-SSPJM2. The dashed line corresponds to the bulk value of $r_{s}$ for cesium (5.63). (b) Same as (a) but for singly ionized cesium clusters.

Figure 3: (a) Same as Fig.2 for neutral Na clusters. The bulk value is 3.99. (b) The average distance between nearest neighbors in atomic units for Na clusters. The squares correspond to our findings through SSPJM2, appropriate for room temperature and the diamonds correspond to the molecular dynamics results at zero temperature. The dotted line correspond to the bulk value 7.07. (c) Same as (a) for singly ionized Na clusters.

Figure 4: (a) The equilibrium $r_{s}$ values in atomic units for neutral Al clusters $\left(z^{*}=1\right.$, $\left.r_{s}=2.07\right)$. The diamonds and squares show the physical points in SSPJM2 and SJM1 schemes, respectively. (b) Same as (a) for singly ionized Al clusters.

Figure 5: (a) The total energies per atom of Na clusters in electron volts for the SSPJM2 and the SSPJM1. The SSPJM2 results are somewhat lower than those of the SSPJM1 for smaller clusters. (b) The ionization energies in electron volts for Na clusters in the dif-SSPJM2 and the dif-SSPJM1 are compared with the experimental values.[9] 


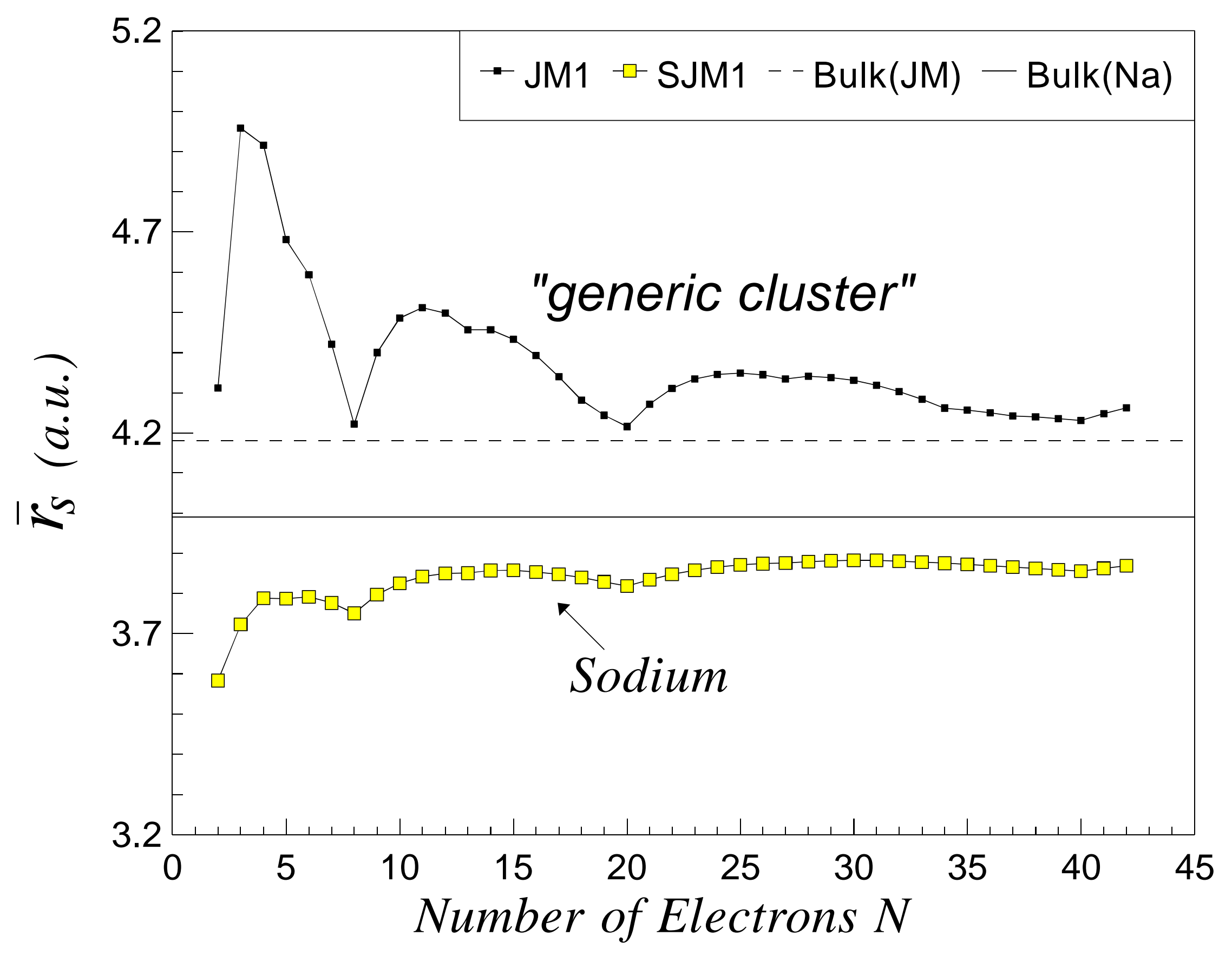




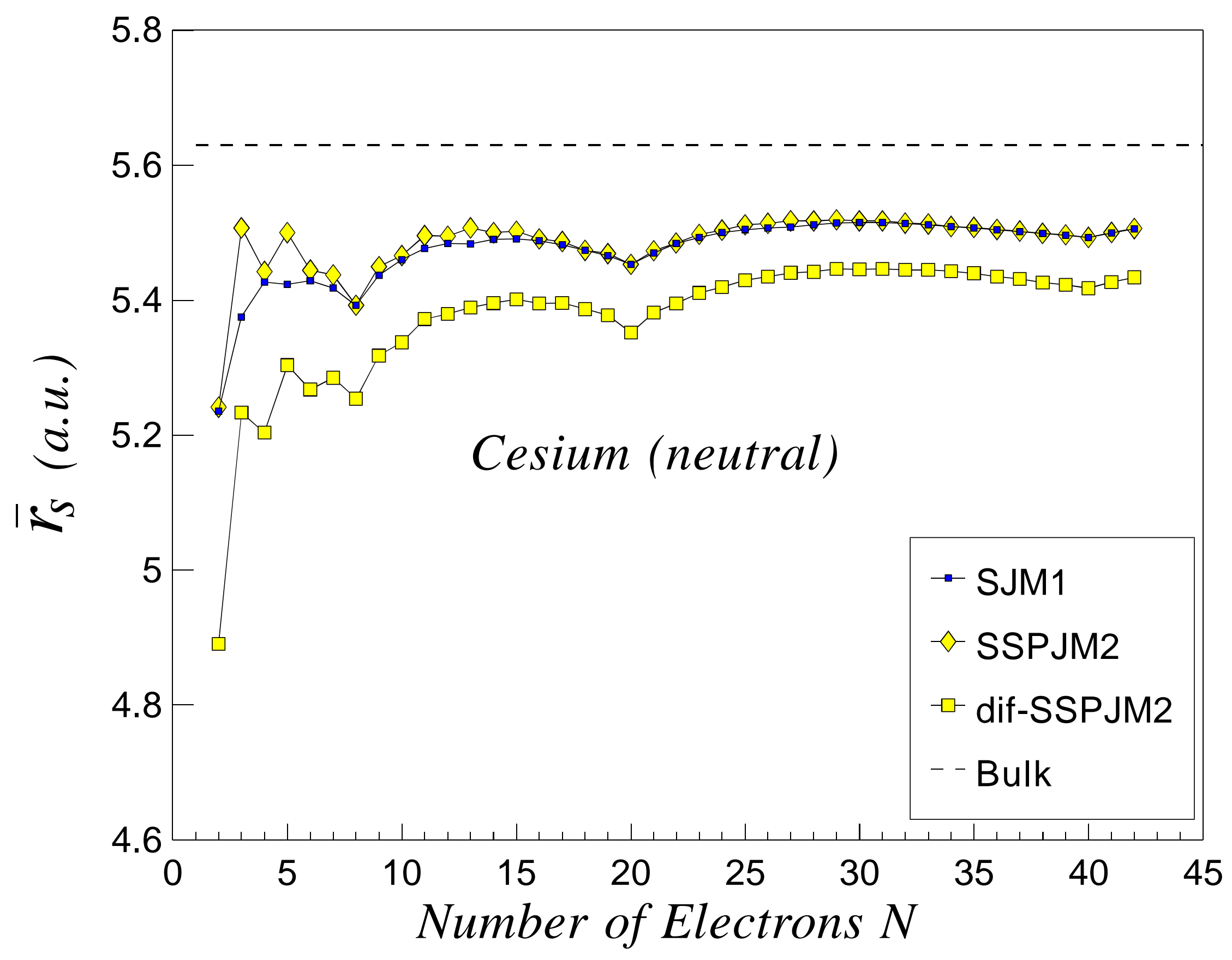




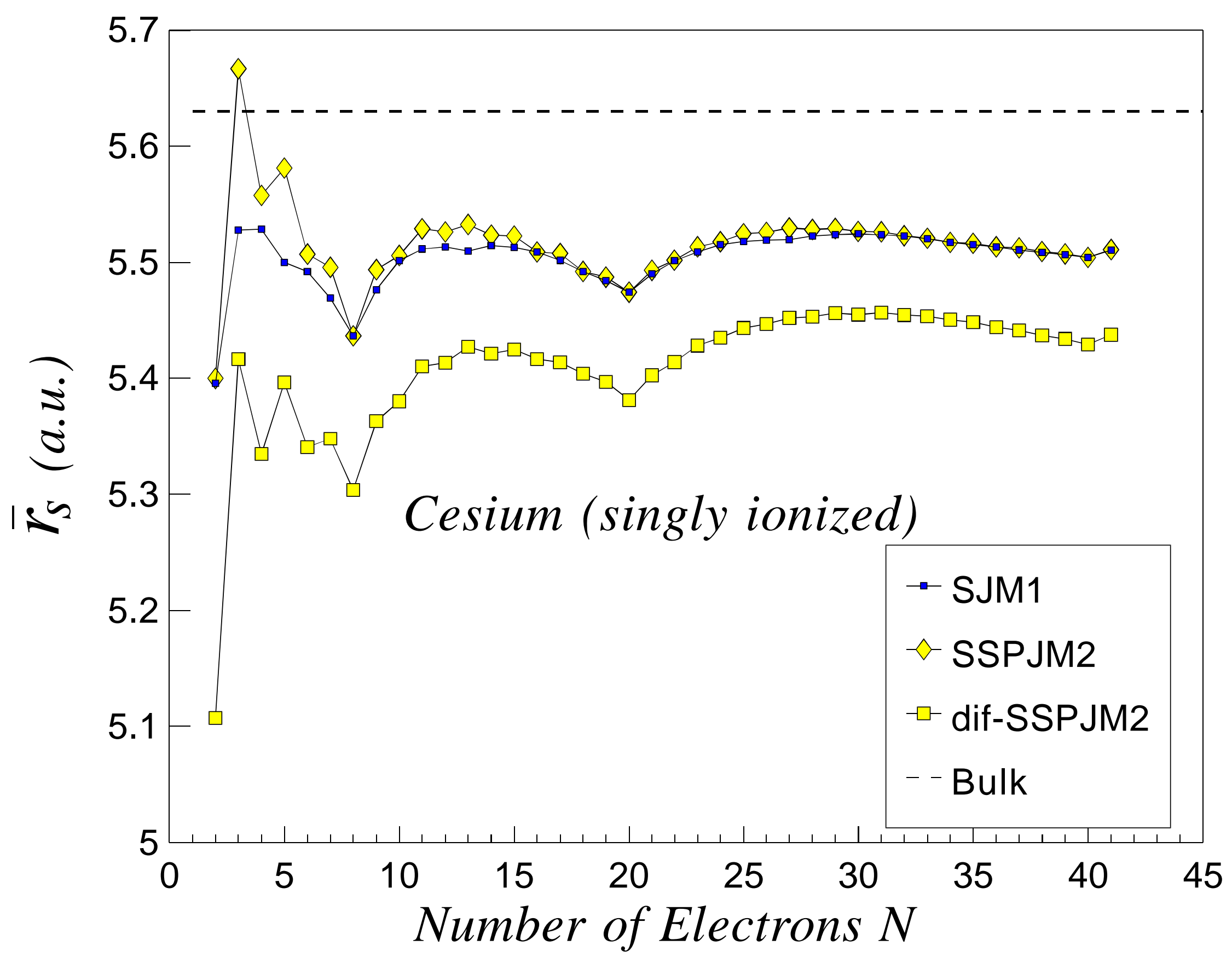




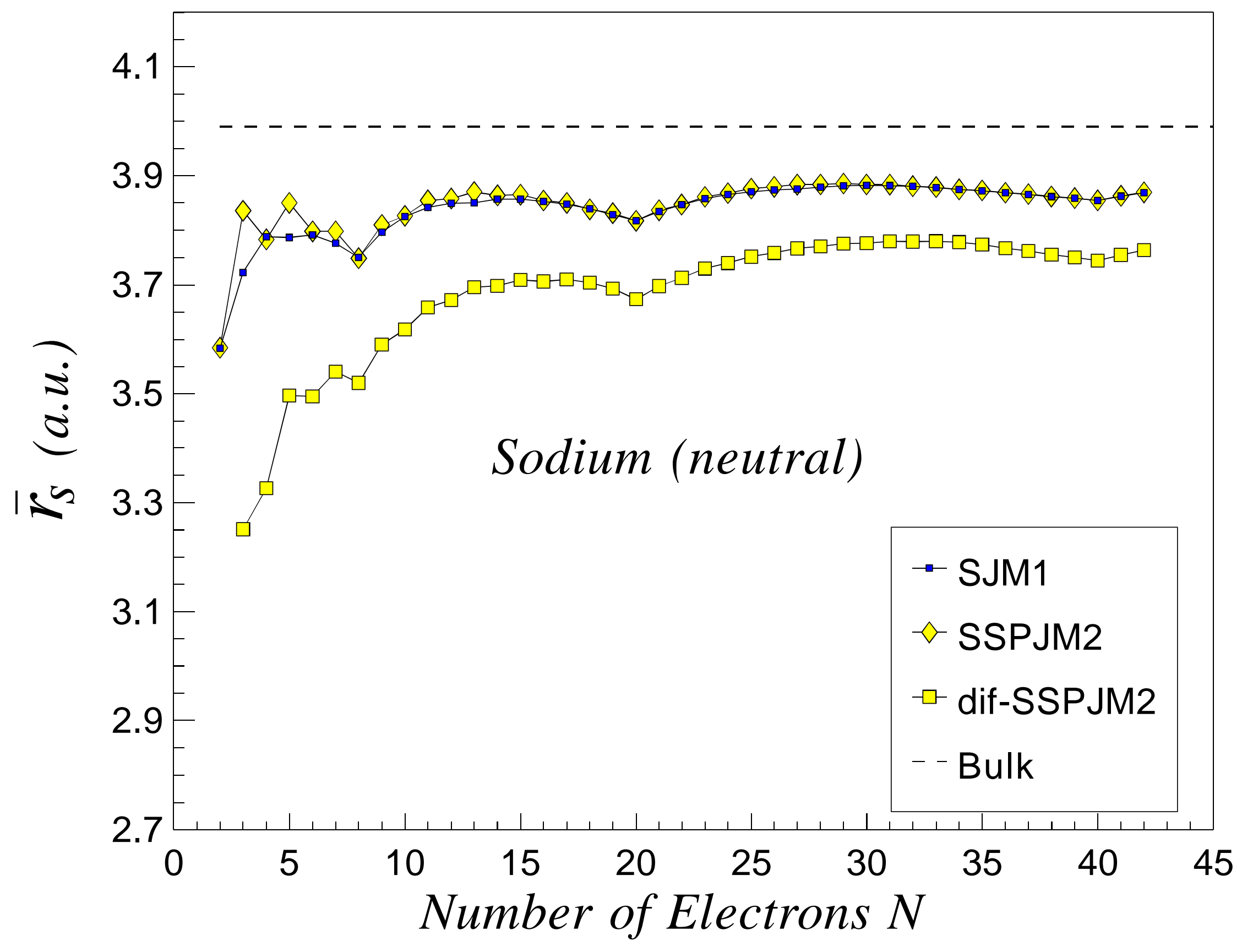




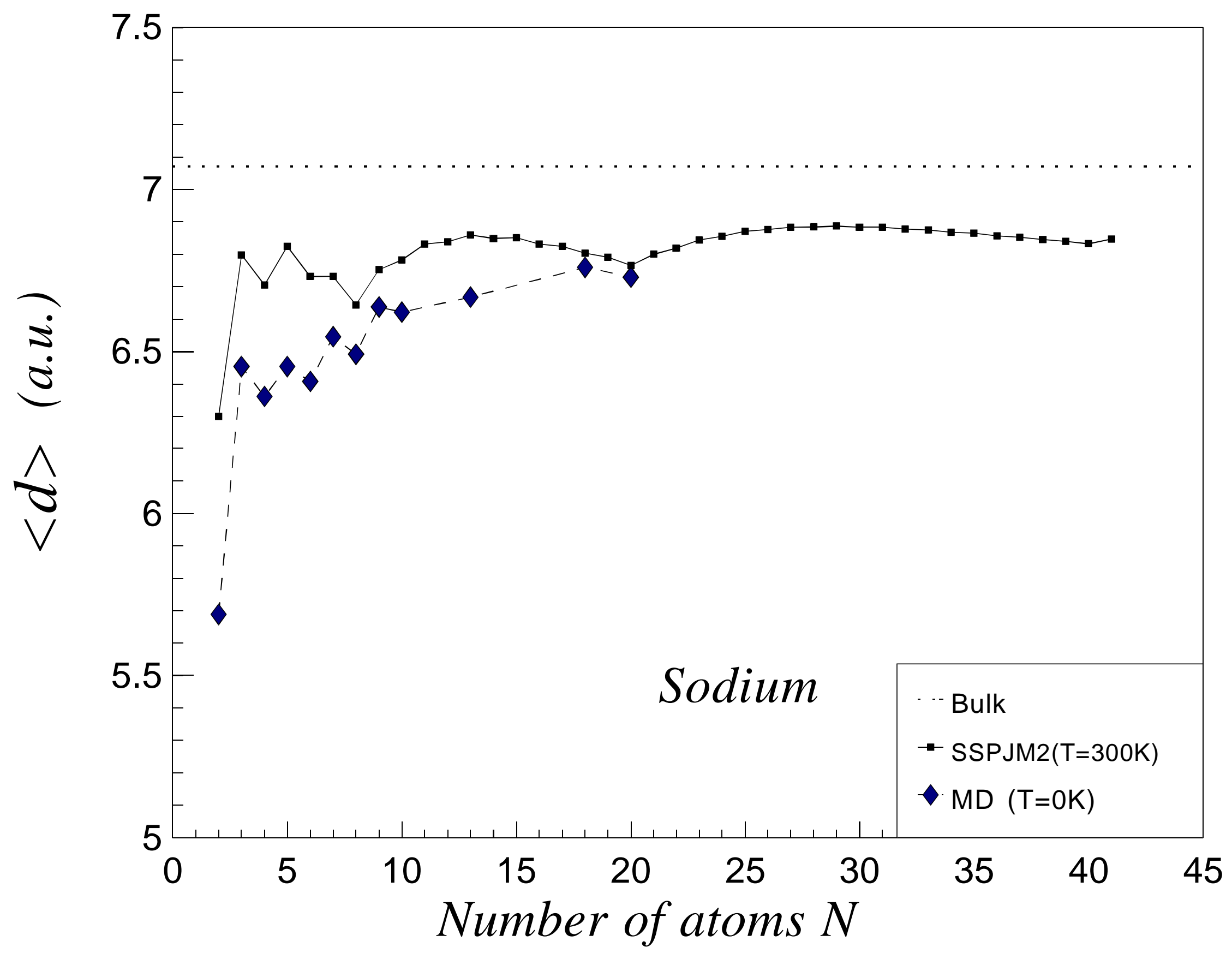




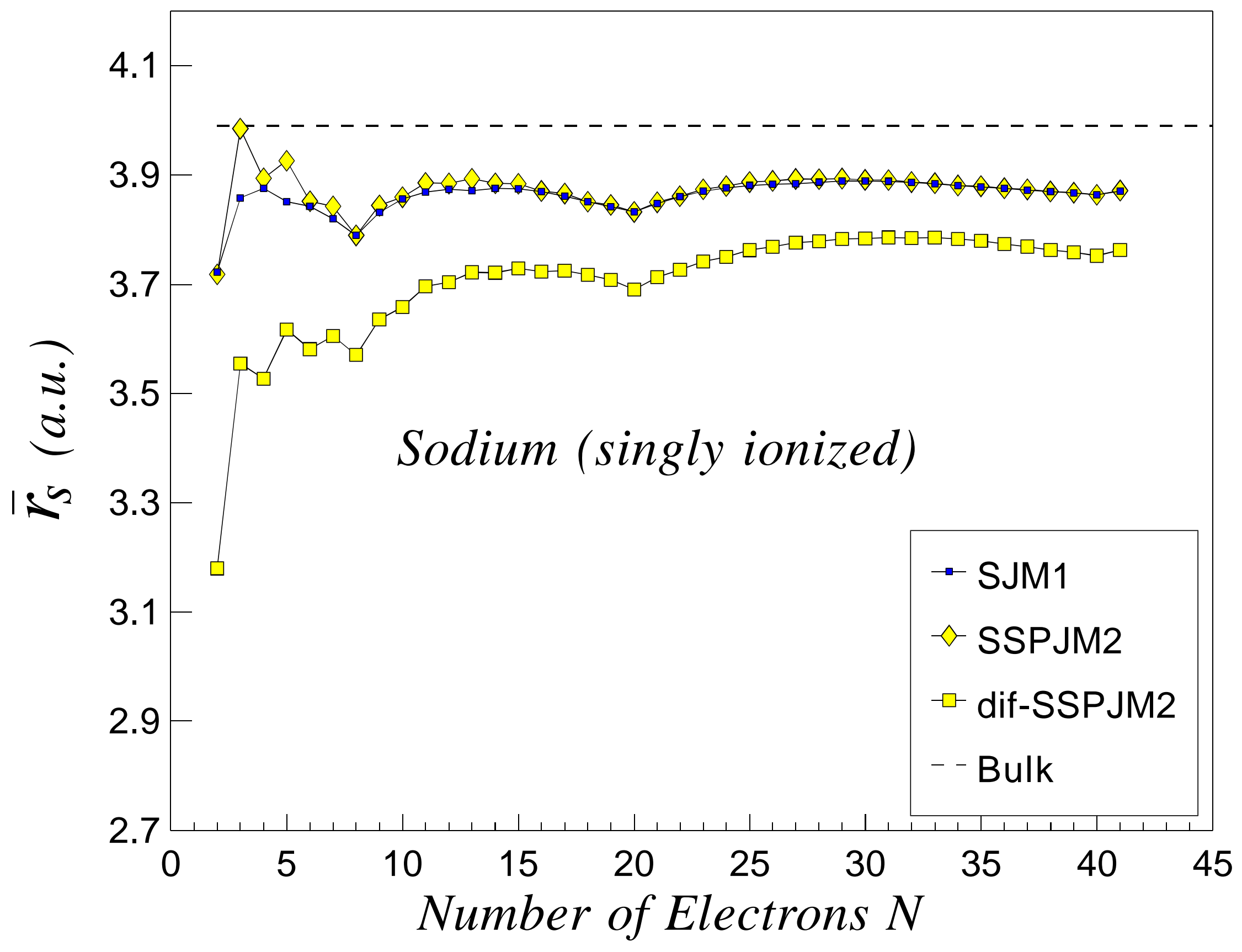




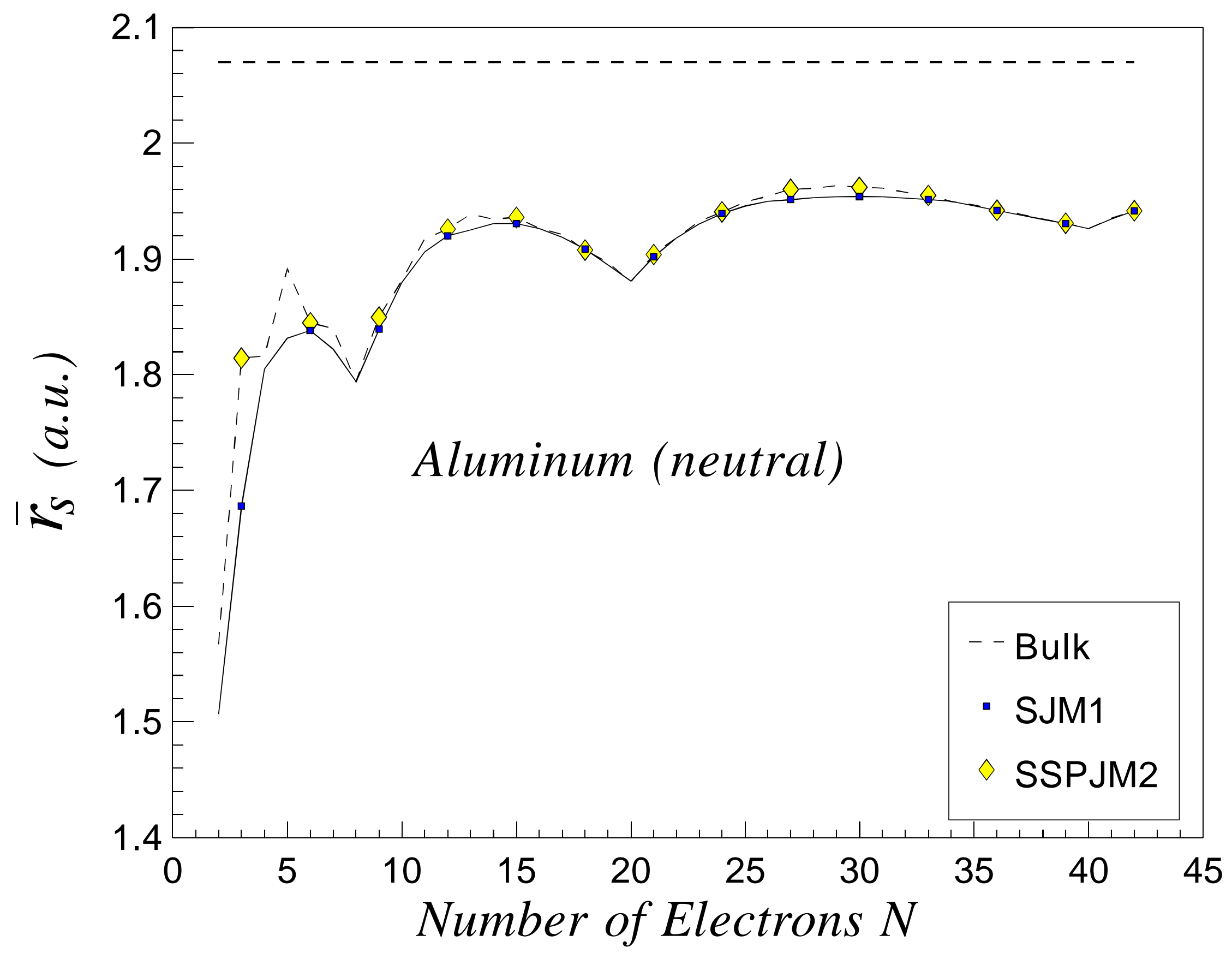




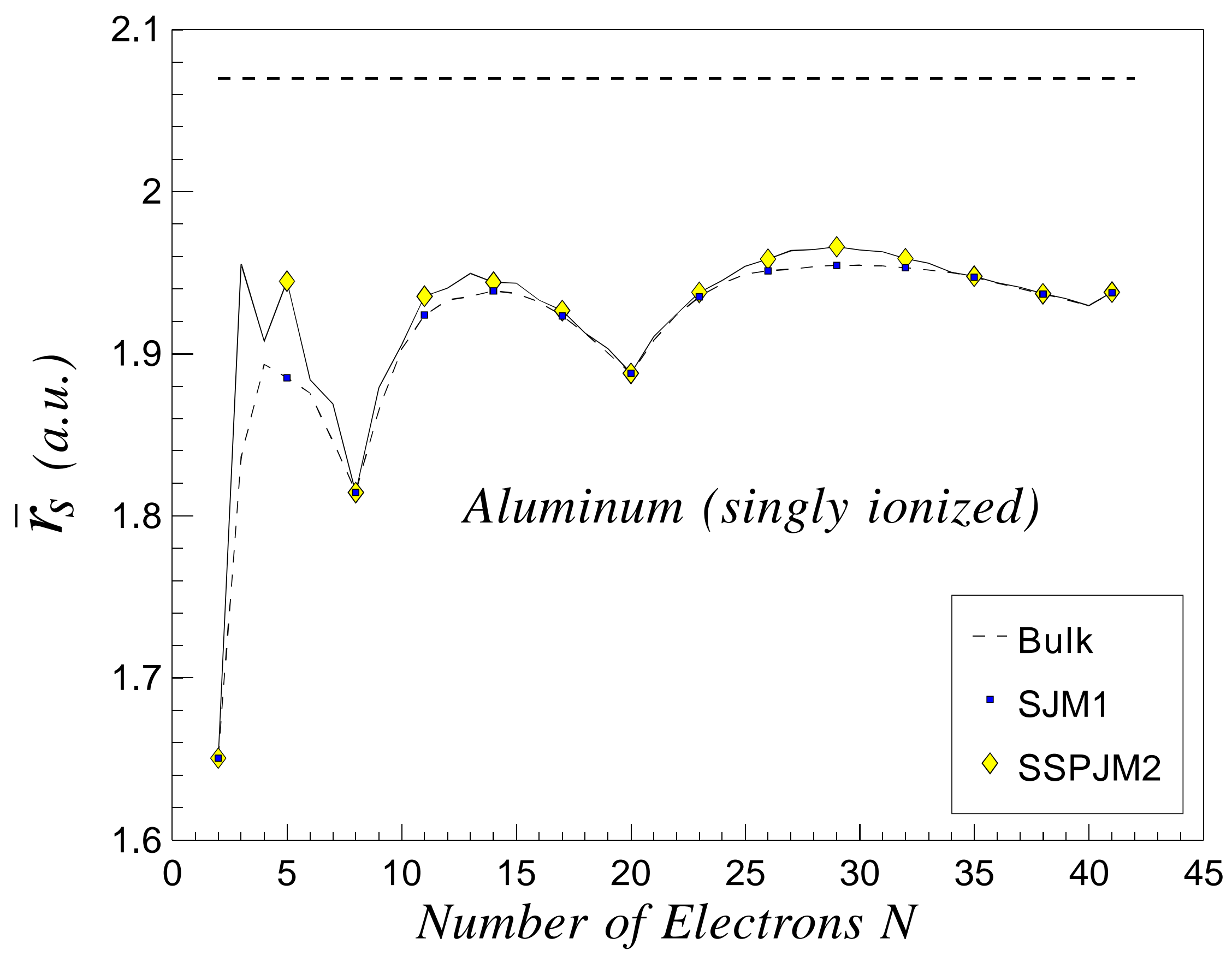




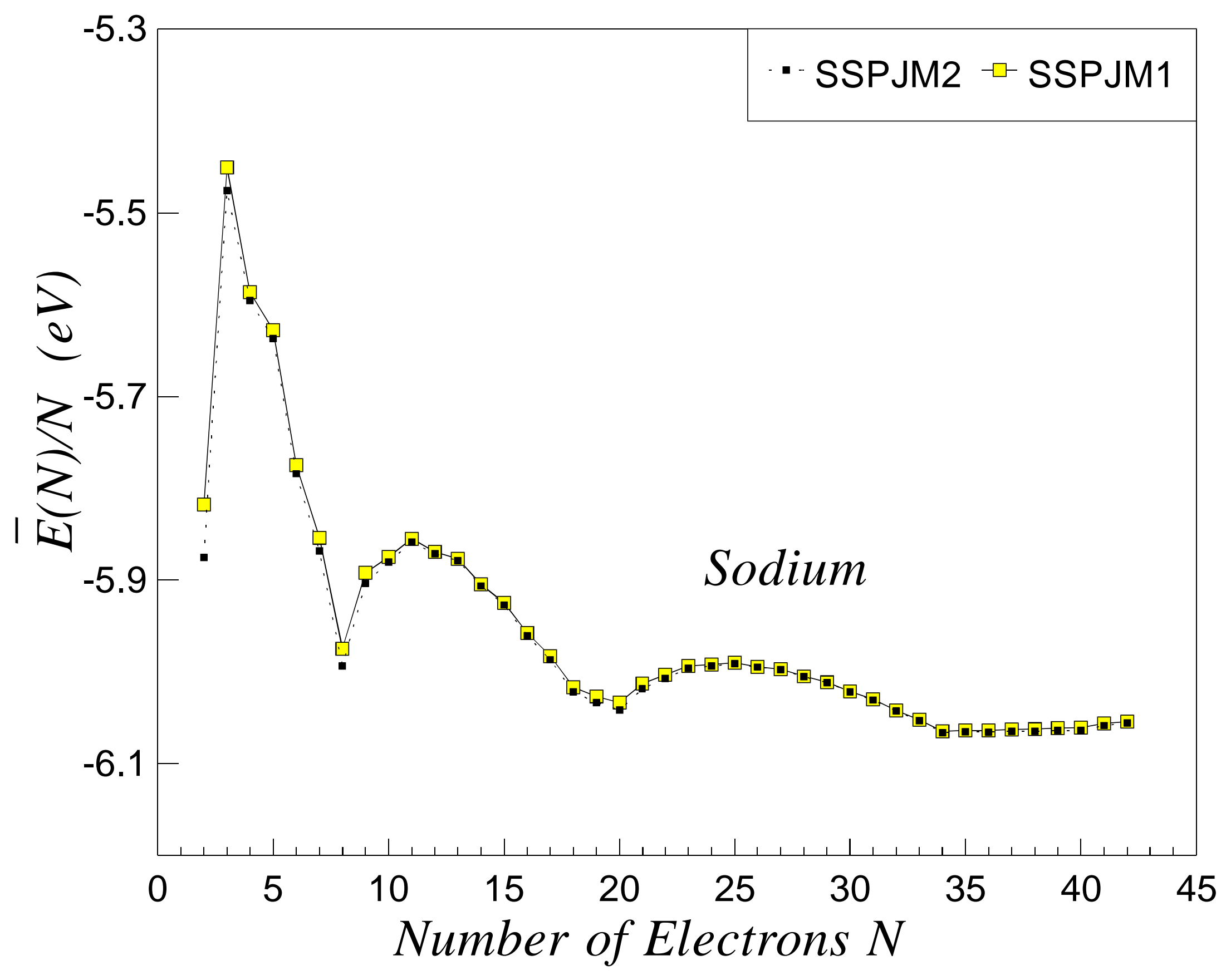




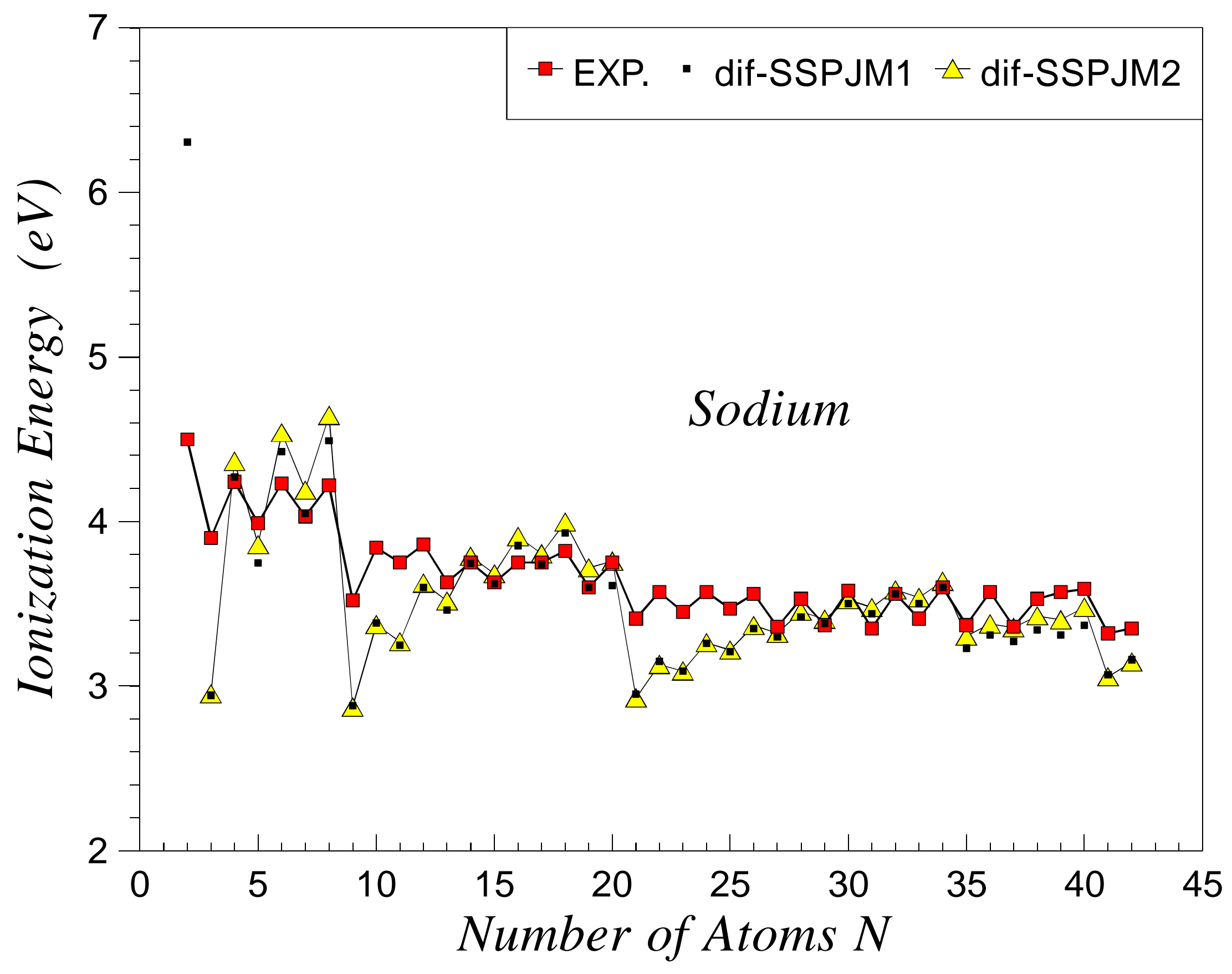

